\title{
Experimental and numerical investigation of mixed flow in a gallery
}

\author{
S. Erpicum ${ }^{1}$, F. Kerger ${ }^{1,2}$, P. Archambeau ${ }^{1}$, B. J. Dewals ${ }^{1,2}$ \\ \& M. Pirotton ${ }^{1}$ \\ ${ }^{I}$ Research Unit of Hydrology, \\ Applied Hydrodynamics and Hydraulic Constructions - HACH, \\ ArGEnCo Department, Liege University, Belgium \\ ${ }^{2}$ Belgian Fund for Scientific Research - FRS-FNRS, Belgium
}

\begin{abstract}
Experimental investigations on a physical model of a gallery performed in the Laboratory of Structures Hydraulics at the University of Liege are presented. The study focuses on the influence of the gallery aeration rate on the mixed flow pattern and the pressure distribution. In particular, the effect of air vents on the flow patterns and the release capacity of the gallery are assessed. An unusual mechanism leading to a two-phase instability is also pointed out when the aeration rate is not sufficient. Experimentations are completed with numerical computations performed with an original $1 \mathrm{D}$ model developed by the authors to give new insight into the mechanisms involved.
\end{abstract}

Keywords: hydraulics, mixed flows, civil engineering, aeration.

\section{Introduction}

Mixed flows, characterized by the simultaneous occurrence of free-surface and pressurized flows, are frequently encountered in rivers networks, sewer systems, storm-water storage pipes, flushing galleries... As a matter of fact, some hydraulic structures are designed to combine free-surface and pressurized sections (e.g. water intakes). Dynamic pipe filling bores may occur in hydraulic structures designed only to convey free-surface flow. During such a transition, highly transient phenomena appears and may cause structural damages to the systems [1], generate geysers through vertical shafts [2] and engender flooding.

What is more, air/water interactions may arise in such structure (particularly at the transition bore [3]) and thoroughly alter the flow regime. Sometimes the 
interactions produce beneficial effects. However, more often than not, the effects are not beneficial and the remedial actions are expensive. For instance, the presence of air in pipelines can severally affect the water carrying capacity of the line, increase head losses, produce undesirable pressure rise, and even induce water hammer or blowbacks by air evacuation [4]. The effect of air in such situations depends on the location and amount of the un-dissolved air as well as the configuration of the hydraulic structure. In addition, the prediction of such flows by means of a numerical scheme remains challenging by the lack of suited mathematical model and closure relations [5].

Traditionally, the common design of hydraulic structures was conducted assuming either stratified or pressurized single phase flow. The need for additional flexibility in the hydraulic electricity production on the one hand and more rigorous restriction due to environmental and political demands on the other hand lead to an increasing demand for further research on air/water interaction for civil engineering applications. As pointed out in [6], multiphase flow in hydraulic structures is a topic of recent interest. In fact, the only comprehensive description of the relevant flow patterns could be found in approaches originating from chemical and process engineering. As the typical internal diameter of the conduits in chemical engineering is at least one order of magnitude smaller than in hydraulic engineering, the application of these concepts to hydraulic works remains challenging.

In this paper, the results of the experimental study of stationary mixed flows taking place in a gallery are presented in detail. Experimental investigations have been carried out on a model of a gallery in the Laboratory of Structures Hydraulics $(\mathrm{HACH})$ of the Liege University. The model includes a Plexiglas pipe linking two tanks. The topography of the upstream and downstream tanks has been built regarding realistic in-situ conditions. The aim of the experimental study was to:

1. Determine the expected flow discharge and the influence of the aeration rate on the flow discharge.

2. Identify hydrodynamic characteristics of the flows appearing in the gallery and appearance conditions for each pattern.

In particular, the experimental apparatus is clearly described in this paper as well as the measurement system and the investigation method.

Finally, numerical simulations have been performed with an original 1D model for mixed flows, developed by Kerger et al. [5]. The model is implemented in the $1 \mathrm{D}$ module of the software package WOLF. WOLF is a finite volume flow simulation modelling system developed within the Laboratory of Hydrology, Applied Hydrodynamics and Hydraulic Constructions (HACH) at the University of Liege. Stationary numerical results give new insight into the mechanisms regulating flows in the gallery.

\section{Experimental set up}

\subsection{Physical model}

The experimental facilities are made of two tanks, an upstream and a downstream one, linked by a circular gallery $5 \mathrm{~m}$ long with a $.14 \mathrm{~m}$ diameter. 
The natural topography of a mountain river bed is represented in both tanks, as the gallery bypasses a river meander. The gallery inlet and outlet are located in the right bank of the river, at the level of the river bottom, and the constant gallery slope is $6.96 \%$. The inlet structures are profiled to decrease the head losses at the gallery entry and a $.17 \mathrm{~m}$ long square to circle transition links the inlet to the gallery. The gallery is not straight but counts for an upstream bend of 27.68 degrees with a curvature radius of $1.81 \mathrm{~m}$ and a sharp angle of 2.5 degrees at its middle (figure 1). The outlet is rectangular with a radial gate $.12 \mathrm{~m}$ high and $.12 \mathrm{~m}$ wide to control the flows through the gallery. A $.17 \mathrm{~m}$ long circle to square transition links the gallery to the outlet. The tanks are made of steel; the tanks topography has been build with concrete blocks and mortar painted with latex. The gallery is in transparent Plexiglas and the inlet and outlet are made of aluminium and PVC. The roughness height of the gallery has been estimated to be $2.10^{-5} \mathrm{~m}$.

In a second part of the study, three air vents with a $.02 \mathrm{~m}$ diameter have been added upstream of the gallery, on the top of the circular cross section, at the beginning of the constant slope. The purpose of these vents is to feed the gallery with air to prevent the formation of low pressures within the flowing water. Air vents have been designed according to the recommendations in [7].

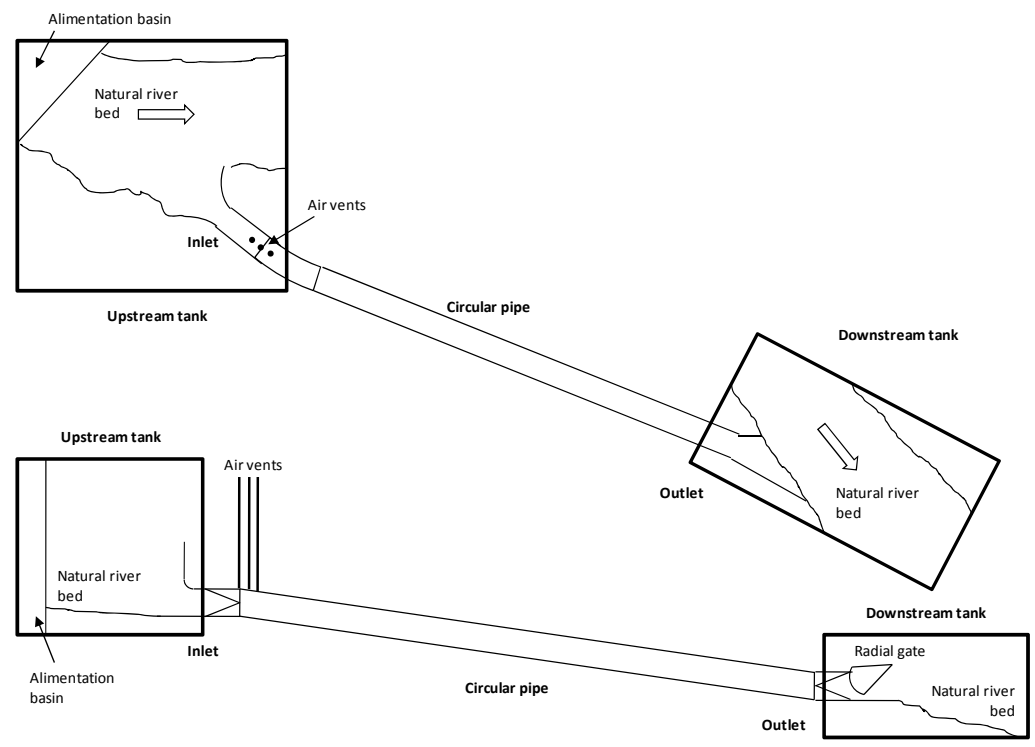

Figure 1: $\quad$ Sketch of the experimental device.

\subsection{Water alimentation and boundary conditions}

The water feeding system is a closed circuit with a pump taking water from a $400 \mathrm{~m}^{3}$ underground reservoir to inject it through under pressure galleries in an alimentation basin in the upstream tank. The alimentation basin is separated from 
the upstream tank by a permeable screen to make uniform the velocity fields entering the physical model. Downstream of the model, the water is collected in a free-surface channel to go back to the underground reservoir.

The discharge in the upstream tank is the upstream boundary condition. The head level upstream of the gallery regulates naturally regarding the gate opening rate and the system release capacity. Downstream of the physical model, the natural topography is very steep so no specific boundary condition is needed (supercritical flow).

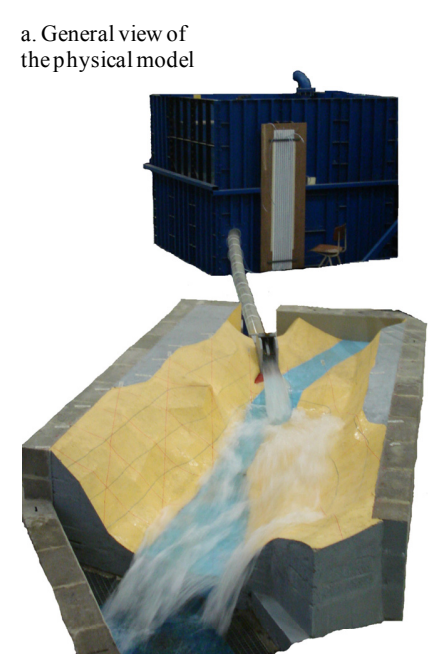

Figure 2: $\quad$ Details of the physical model.
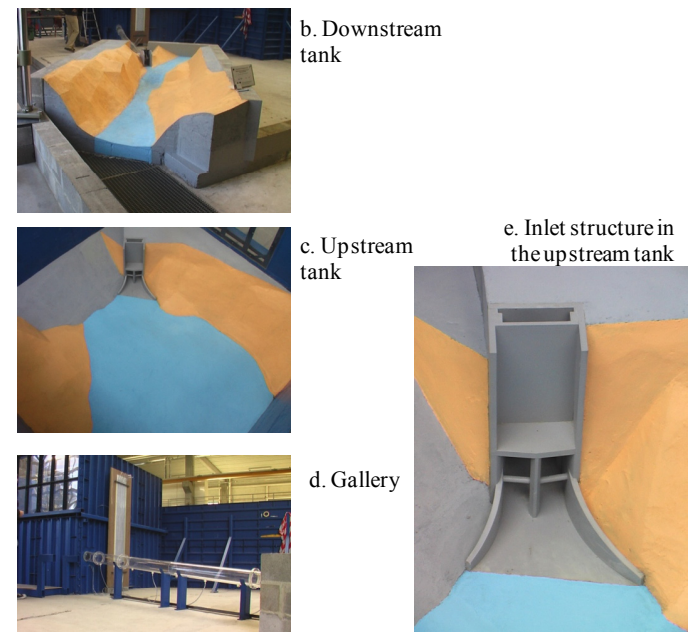

e. Inlet structure in the upstream tank

\subsection{Measurement system}

The model is equipped with the following measurement system:

- The upstream discharge is measured with an electromagnetic dischargemeter (accuracy of $\pm 11 / \mathrm{s}$ ) on the pumping system;

- The water level in the upstream tank is measured using a limnimeter (accuracy of $0.1 \mathrm{~mm}$ ) and a Pitot tube (accuracy of $\pm 0.1 \mathrm{~mm}$ );

- 9 Pitot tubes are regularly distributed along the gallery to measure the pressure head in the gallery (accuracy of $\pm 0.1 \mathrm{~mm}$ );

- 14 graduated scales are fixed on the gallery perimeter to measure the water level for stratified flows.

\section{Numerical model}

In the last decades, large literature $[8,9]$ has been dedicated to a new approach in experimental hydraulics consisting in coupling experimental investigations with CFD computations. Comparison of the experimental data with numerical results 
from a modelling system is shown to give new insight into the flow conditions and to significantly reduce the cost of the physical models.

The numerical model used in this paper is an original 1D model for mixed flows developed by Kerger et al. [5]. It is implemented in the one-dimensional module of the modelling system WOLF. WOLF is finite volume scheme developed within the Laboratory of Hydrology, Applied Hydrodynamics and Hydraulic Constructions (HACH) of the University of Liege [10].

WOLF 1D is based on a set of hyperbolic Partial Differential Equations (PDE), usually called Saint-Venant equations [11], describing one-dimensional unsteady open channel flow. The Saint-Venant equations are derived from cross section integration of the Navier-Stokes equation:

$$
\frac{\partial}{\partial t}\left(\begin{array}{l}
\mathrm{A} \\
\mathrm{Q}
\end{array}\right)+\frac{\partial}{\partial \mathrm{x}}\left(\begin{array}{c}
\mathrm{Q} \\
\mathrm{Q}^{2} / \mathrm{A}
\end{array}\right)=\left(\begin{array}{c}
0 \\
\mathrm{gA}\left(\frac{\partial \mathrm{Z}}{\partial \mathrm{x}}-\mathrm{S}_{\mathrm{f}}\right)
\end{array}\right)
$$

where $\mathrm{A}\left[\mathrm{m}^{2}\right]$ is the cross section, $\mathrm{Q}\left[\mathrm{m}^{3} / \mathrm{s}\right]$ is the flow discharge, $\mathrm{g}\left[\mathrm{m}^{2} / \mathrm{s}\right]$ is the gravity, $Z[\mathrm{~m}]$ is the free surface elevation, $\mathrm{S}_{\mathrm{f}}[-]$ is the friction slope resulting from the resistance law. Friction slope $S_{F}$ may be computed using the DarcyWeisbach relation and the Colebrook relation for the friction factor:

$$
S_{F}=\frac{f u^{2}}{2 D_{h}} \text { with } \sqrt{\frac{1}{f}}=-2 \log \left(\frac{k}{3.7 D_{h}}+\frac{2.51}{\operatorname{Re} \sqrt{f}}\right)
$$

with $\mathrm{D}_{\mathrm{h}}[\mathrm{m}]$ the hydraulic diameter of the cross-section, $\mathrm{k}[\mathrm{m}]$ the roughness height, $\mathrm{u}[\mathrm{m}]$ the water velocity and Re[-] the Reynolds Number.

Pressurized flows are commonly described through the Allievi equations [12]. According to the Preissmann slot model [13], pressurized flow can equally be calculated through the Saint-Venant equations by adding a conceptual slot on the top of a closed pipe [5]. When the water level is above the cross section maximum level, it provides a conceptual free surface flow, for which the gravity wave speed is $c=\sqrt{g \Omega / T_{f}}$ ( $\mathrm{T}_{\mathrm{f}}$ is the slot width). Physically, the slot accounts naturally for the water compressibility and the section dilatation under a variation of pressure. In order to simulate pressurized flows with a piezometric head below the top of the pipe section, an original concept, called negative Preissmann slot [5], has been developed.

\section{Results}

Investigations focused mainly on stationary flows and aimed at determining the flow discharge as a function of the upstream pressure head and downstream gate opening. In this case, strong air/water interactions alter the flow behaviour. In particular, the flow discharge through the gallery is strongly influenced by air/water interaction and depends of the aeration rate. In this section, the influence of both the upstream pressure head and the aeration conditions on the release capacity of the gallery is analyzed. 


\subsection{Without air vents}

Figure 4 shows the experimental relation between the flow discharge through the gallery and the upstream pressure head (zero level is set at the upstream reservoir bottom level) without air vents. Various two-phase flow patterns are observed according to the flow discharge. 5 areas corresponding to the 5 flow patterns that are usually mentioned in literature [14] can be defined. Details of each flow pattern are provided on figure 3 :

1. Pure water fully free surface flow (or smooth stratified flow) is observed for pressure head below $30 \mathrm{~cm}$ (figure 3.a). It is characterized by both phases, air and water, flowing separately by gravity. A smooth interface between phases appears only if both phases flow with almost the same velocity.

2. Wavy stratified flow is observed for pressure head between $30 \mathrm{~cm}$ and $40 \mathrm{~cm}$ (figure 3.b). This flow does not differ much from smooth stratified flow in terms of hydro-mechanic characteristics. Surface ripples and waves are building up and create a rather rough interface. The volume of airflow, entering by inlet as dissolved air in water and through a vertical vortex appearing at the gallery intake, is equal to the air volume insufflated into the flow by self-aeration plus the air volume flowing above the water surface as a result of the air-water shear forces.

3. Intermittent flow is observed for pressure head between $40 \mathrm{~cm}$ and $55 \mathrm{~cm}$. This latter category includes slug flow (figure 3.c), where waves touch the top of the tube and form a liquid slug which passes rapidly along the gallery; as well as plug flow (figure 3.d), in which there are large bubbles flowing near the top of the tube. As the transition from plug to slug flow is gradual and not very sharp, slug and plug flow together are often simply referred to as intermittent flow patterns with no further specification. This flow pattern is not strictly speaking a steady flow as it is characterized by a time periodic oscillation between a free surface flow and slug flow, according to the aeration rate and the amplitude of the waves. This leads to significant fluctuations in the measured data.

4. Bubbly flow, characterized by the entrainment of small bubbles dispersed in the liquid continuum, is observed for pressure head between $55 \mathrm{~cm}$ and 70 $\mathrm{cm}$ (figure 3.e). The larger bubbles (but smaller than the resulting pockets during plug flow) propagate below the conduit ceiling due to buoyancy. Smaller bubbles, primarily transported by liquid turbulence, may be detected dispersedly over the whole cross-section. Bubbles appear where the pressure of the liquid falls below the atmospheric pressure.

5. A pure water pressurized flow is observed for pressure head above $70 \mathrm{~cm}$ (figure 3.f).

Two curves computed with WOLF are represented on the graph of figure 4. The dotted line is computed assuming that a free surface appears if the water height is below the cross section top (air phase above the free surface is at atmospheric pressure - high aeration rate). The continuous line is computed by activating the negative Preissmann slot (sub-atmospheric pressurized flow - low aeration rate). 
a. Smooth Stratified flow
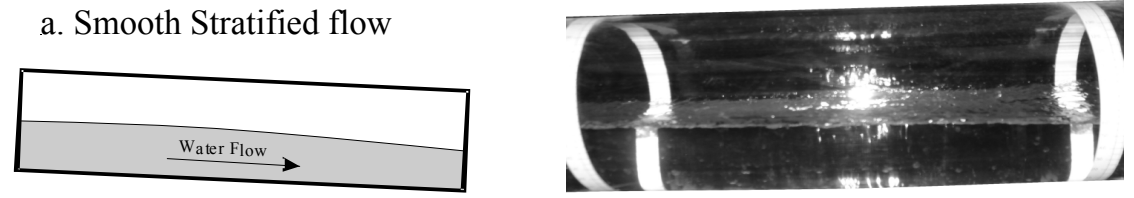

b. Wavy Stratified flow
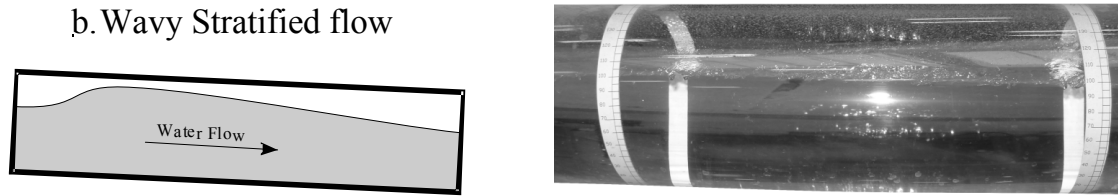

c. Intermittent - Slug flow
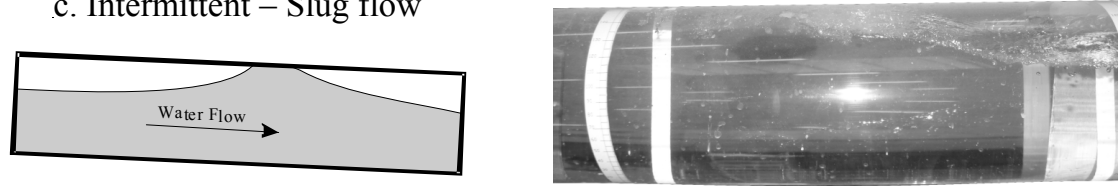

d. Intermittent - Plug flow
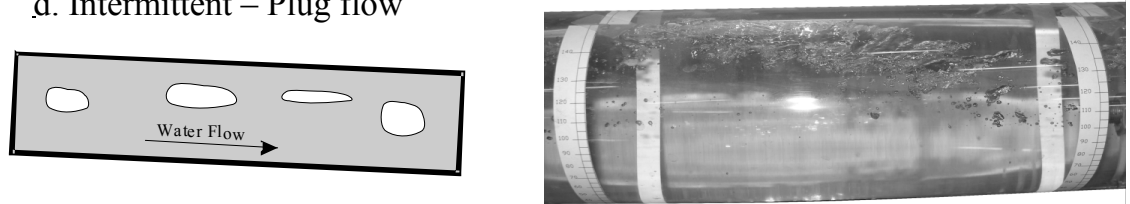

e. Bubbly flow
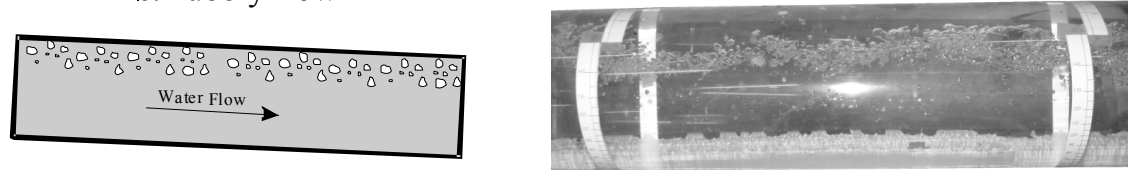

f.Pressurized flow

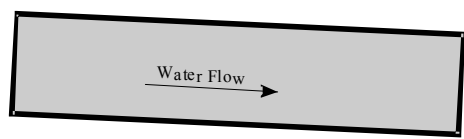

Figure 3: $\quad$ Flow patterns visualization.

Numerical results are in good accordance with experimental data for smooth stratified flows and fully pressurized flows. Bubbly and intermittent flows show a similar behaviour to the sub-atmospheric pressurized flows. This point underlines the aeration rate in the gallery is too small to create a free surface flow. 


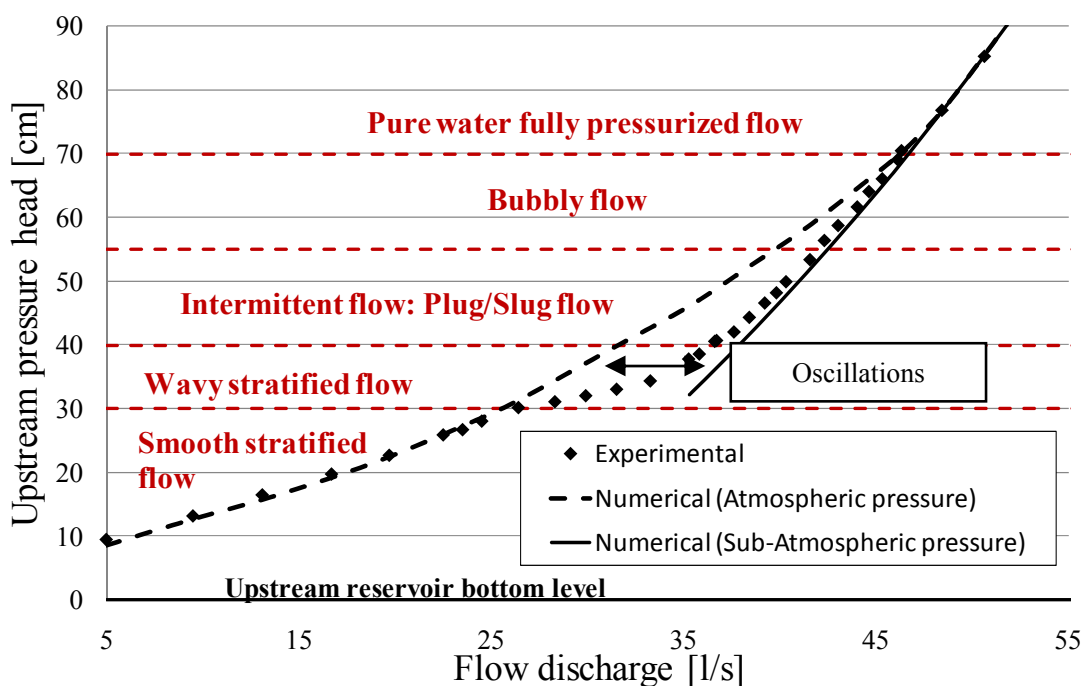

Figure 4: Experimental relation (upstream pressure head-flow discharge) and flow patterns observed.

\subsection{Periodic instabilities}

A periodic instability between two unstable steady flow regimes occurs in the area denoted by wavy stratified flows (figure 4). The instability induces large period (10 to 60 seconds) oscillations of the water level in the upstream reservoir. The amplitude of the oscillations can reach $2 \mathrm{~cm}$. In addition, pressure oscillations, whose amplitude can reach $4 \mathrm{~cm}$, are observed all along the gallery.

The inception of the instability is intimately linked with the aeration rate of the gallery, and in particular the amount of air entrained through the vertical vortex appearing at the water intake. Indeed, the minimum of the oscillation corresponds to a highly aerated stratified flow below an air phase at subatmospheric pressure. The distribution of pressure along the gallery in this case is given in figure 5.b. As pointed in figure 4, this flow pattern gives the minimum water carrying capacity for a given upstream head. If the upstream reservoir is supplied with a constant flow discharge, the water level in the reservoir increases.

As the water level arises in the reservoir, the amount of air entrained through the vortex decreases [15]. Decrease of the air void fraction in the flow generates the formation of Kelvin-Helmholtz instabilities characteristic of intermittent flows (pressurized flow pattern). The maximum of the oscillations corresponds to this poorly aerated intermittent flow. The distribution of pressure along the gallery in this case is given in figure 5.a. As pointed in figure 4, this flow pattern gives the maximum water carrying capacity for a given upstream head. Then, the water level in the reservoir decreases because the flow discharge in the gallery is higher than the flow discharge supplying the reservoir. The cycle of the instability is then explained. 

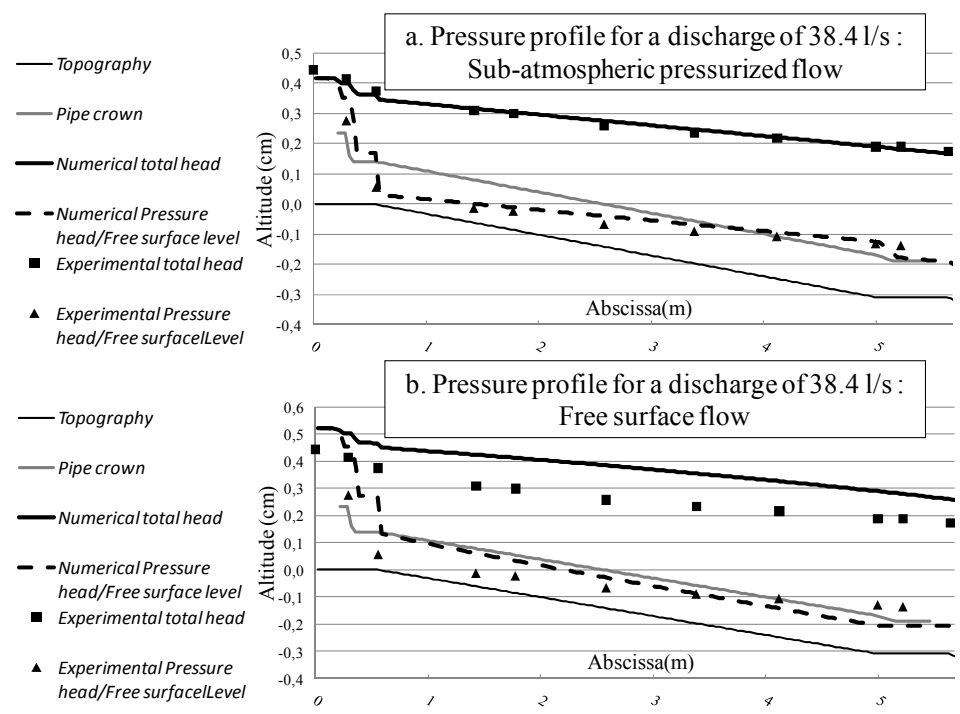

Figure 5: Computed total head and pressure head distribution for an intermittent flow sub-atmospheric pressurized flow and freesurface flow computation.

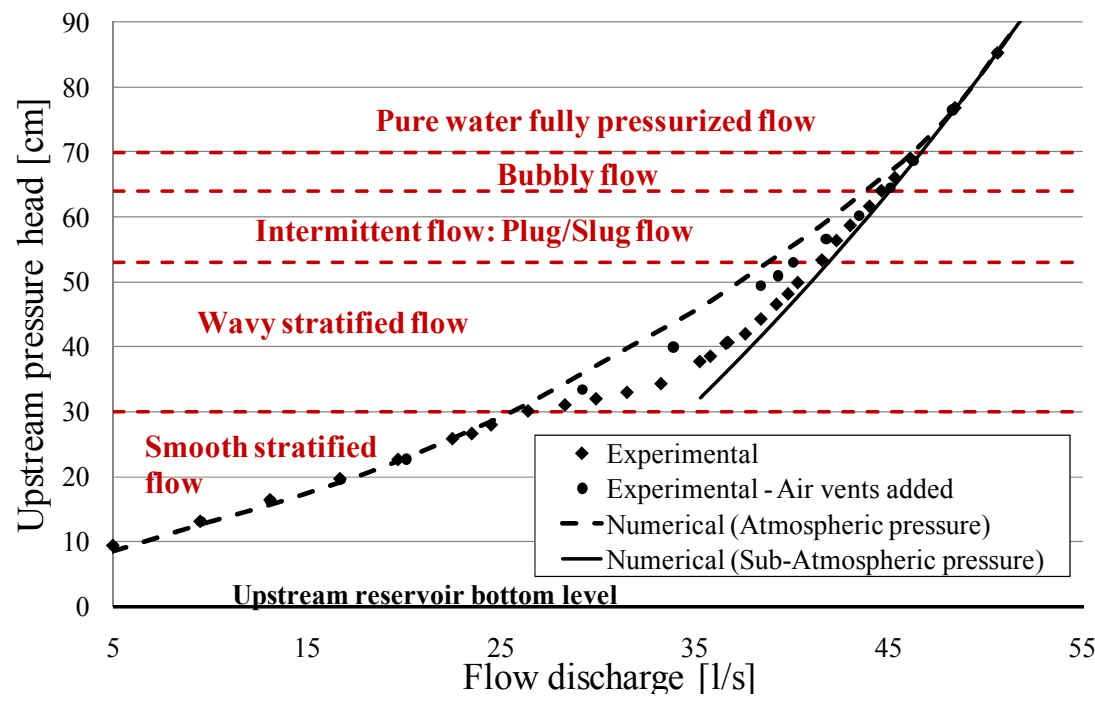

Figure 6: Effects of the addition of air vents on the experimental relation head/discharge and the flow pattern observed. 


\subsection{Air vents effect}

In this section, the effect of the 3 air vents added upstream of the gallery, on the top of the circular cross section, at the beginning of the constant slope is assessed. The purpose of these vents is to enable air to enter the gallery to prevent the formation of low pressures within the flowing water (figure 5.a). The experimental relations between the flow discharge through the gallery and the upstream pressure head is shown on figure 6.

The graph compares the new relation with the relation without air vent. Addition of air vents does not affect pure water flow, as expected. On the opposite, a diminution of the water carrying capacity of the gallery is observed for multiphase flow patterns. Area of observation of each two-phase flow pattern is affected as well. The ranges of flow discharge corresponding to bubbly flows and corresponding to intermittent flows are narrowed to the benefit of the wavy stratified flow area.

\section{Conclusions}

Experimental investigations on a physical model of a gallery have been presented. They were completed with CFD computations to give more insight into the mechanisms involved. Flow patterns description and mathematical models originating from chemical/process engineering served as a referential basis. The key issue of the present work is to show concepts develop for small size pipe can be adapted for conduit sizes significantly larger.

Flow pattern identification has been performed for various aeration rates. In particular, the effect of the aeration rate and the flow pattern over the water carrying capacity has been assessed. Particular insight has been given on the nature of the transition between free surface and pressurized flows. Finally, a two-phase instability has been outlined.

The fundamental concepts introduced pave the way for further research. Experimental research is required to derive air entrainment predictive relation and flow pattern maps more specific to hydraulic structures.

\section{Acknowledgement}

A part of the experimental results is the property of EDF-CIH. The HACH gratefully acknowledge EDF-CIH for the authorization to publish those results.

\section{References}

[1] Zhou, F., F.E. Hicks, and P.M. Steffler, Transient Flow in a Rapidly Filling Horizontal Pipe Containing Trapped Air, Journal of Hydraulic Engineering, 128(6): p. 625-634, 2002

[2] Guo, Q. and C. Song, Dropshaft Hydrodynamics under Transient Conditions, Journal of hydraulic Engineering, 117(8): p. 1042-1055, 1991 
[3] Vasconcelos, J. and S. Wright, Experimental Investigation of Surges in a Stormwater Storage Tunnel, Journal of hydraulic Engineering, 131(10): p. 853-861, 2005

[4] Estrada, O.P., Investigation on the Effects of Entrained Air in Pipelines, in Eigenverlag des Instituts für Wasserbau der Universität Stuttgart. 2007, Universität Stuttgart. p. 200.

[5] Kerger, F., S. Erpicum, P. Archambeau, B.J. Dewals, and M. Pirotton. Numerical Simulation of 1D Mixed Flow with Air/Water Interaction in Multiphase Flow New Forest, 2008

[6] Keller, U., in Versuchanstalt für Wasserbau, Hydrologie und Glaziologie der Eidgenössichen. 2006, ETH Zürich: Zürich. p. 250.

[7] Falvey, H.T., Air-Water Flow in Hydraulic Structures. Engineering Monogaph. Vol. 41: United States Department of the Interior. 1980

[8] Dewals, B.J., S. Andre, M. Pirotton, and A. Schleiss. Quasi 2D-numerical model of aerated flow over stepped chutes. in 30th IAHR Congress, Greece, 2003

[9] Erpicum, S., P. Archambeau, B.J. Dewals, S. Detrembleur, A. Lejeune, and M. Pirotton. Interactions between Numerical and Physical Modelling for the design and Optimization of Hydraulic Structures - Example of a Large Hydroelectric Complex. in International Symposium on Hydraulic Structures, XXII Congresso Latinoamericano de Hidrahulica, Ciudd Guayana, Venezuela, 2006

[10] Dewals, B.J., S. Erpicum, P. Archambeau, S. Detrembleur, and M. Pirotton, Depth-Integrated Flow Modelling Taking into Account Bottom Curvature, Journal of Hydraulic Research, 44(6): p. 787-795, 2006

[11] Cunge, J.A., F.M. Holly, and A. Verwey, Practical Aspects of Computational River Hydraulics. [Monographs and surveys in water resources engineering], 3. Boston: Pitman Advanced Pub. Program. 1980

[12] Wylie, E.B. and V.L. Streeter, Fluid transients. Première ed, ed. M.-H. Inc., 385,1978

[13] Preismann, A. Propagation des intumescences dans les canaux et rivieres. in First Congress of the French Association for Computation, Grenoble, France, 1961

[14] Wallis, G.B., One-dimensional Two-phase Flow, ed. M.-H.B. Company. 410, 1969

[15] Quick, C.M., Efficiency of Air-Entraining Vortex Formation at Water Intake, Journal of the Hydraulics Division, Proceedings of the American Society of Civil Engineers, 96(7): p. 1403-1416, 1970 